\title{
Red and Black Pigmentation developing during Treatment of Leprosy with 'B 663'
}

\author{
S. G. BROWNE, M.D., F.R.C.P., F.R.C.s., D.T.м. \\ Leprosy Service Research Unit, Uzuakoli, Eastern Nigeria
}

The changes in skin colour remarked during treatment with B 663 of patients with lepromatous (26) or borderline (2) leprosy, have been briefly referred to in published reports (Browne and Hogerzeil r $962 \mathrm{a}, \mathrm{b})$. This paper records in greater detail certain features both of the ruddiness and of the black coloration. Apart from the obvious bearing of this pigmentation on the acceptability of B $66_{3}$, its development presents features of some pathological interest.

It is known from animal experiments that the safranin dyes in general stain the tissues yellow or orange or red, and the aposafranine, B 663 (2 - $p$ - chloroanilino - 5- $p$ - chlorophenyl - 3:5 dihydro-3-isopropyliminophenazine) is no exception, as Barry and his co-workers have pointed out in their series of carefullydocumented papers (Barry et al. I957, I960; Conalty and Jackson i $962 a$, b; etc.). Allday and Barnes had earlier (I952) reported that another safranin dye, B 283, coloured the skin of leprosy patients red and rendered the urine port-wine in colour; they attributed the latter to a metabolite of anilino-aposafranine (B 283).

In addition to the ruddiness of the tissues, (orange subcutis, pink small intestine, red fur), Chang (I962) noted a dark-blue coloration, 'resembling cyanosis', of the hairless areas (i.e. mouth, ears, tail and lower limbs), which appeared in all animals treated for nine months with $\mathrm{B} 663$. The longer the treatment was continued, the deeper the coloration became: its appearance could be postponed or its intensity diminished by the concurrent administration of isoniazid. Conalty and Jackson ( I962 a) also noted that the skin of their animals, initially pink from the accumulation of B 663 in the tissues, gradually became purplish black as treatment was continued.

THE RED GOLORATION

The skin of all the 28 deeply-pigmented Nigerian patients receiving B 663 developed some degree of ruddiness, the intensity of which was initially obscured to some extent by the depth of natural pigmentation.

\section{Onset}

In some of the lighter-hued patients, slight ruddiness could be detected as early as the tenth day of treatment. In all, it was definitely appreciable early in the second month. With a wide range of natural pigmentation and the gradual appearance of the redness, it was not possible to correlate either the appearance of detectable ruddiness or its subsequent intensity with the dose/body-weight ratio.

\section{Clinical features}

One patient complained of slight skin irritation preceding the ruddiness, but had no accompanying eruption, papular, macular or urticarial. Some patients had some feeling of tension in the succulent infiltrated lesions on the face. Otherwise, the coloration developed without symptoms. The patients volunteered that they experienced a sense of well-being.

The redness was first apparent and subsequently most noticeable in the thin, soft, hairless skin of the face (i.e. the peri-orbital and peri-nasal regions particularly) and the thick, hairless, hypopigmented skin of the palms and soles. Afterwards, the entire skin of the lighterhued could be seen to be ruddy. The conjunctivae were only slightly affected, as was the buccal mucosa.

Lepromatous lesions, including discrete lepromatous nodules and diffusely infiltrated skin, were not affected noticeably more than adjacent skin.

The ruddiness deepened while B 663 was being taken, i.e. for six or 12 months. It began gradually to disappear after the drug was stopped. The speed of disappearance varied 
between individual patients. In general, its intensity decreased during the second month after treatment with B 663 ceased, but the periorbital skin remained somewhat redder than normal for some months.

There was some red staining of undergarments and bedding, which could be minimized by a daily bath.

\section{Urinary findings}

The urine was deep red throughout this period, but no other abnormality, chemical or cellular, could be detected in it by the usual methods of examination.

\section{THE BLACK COLORATION}

During the second and third months of treatment with B 663, all the patients developed some degree of darkening of the skin, particularly of the discrete and diffuse leprosy lesions, but in many patients the entire skin eventually became affected.

A precocious indication of this change was the appearance of a circumscribed area of hyperpigmentation corresponding to the elastoplastcovered site of a biopsy incision. Patch tests for picric acid (which had been used for sterilizing the skin) and for elastoplast were both moderately positive, and the areas tested became hyperpigmented following a localized papular dermatitis. The entire skin of this patient subsequently became very dark.

\section{Clinical features}

The blackish-brown or purplish-black coloration deepened rapidly, and persisted unchanged for several weeks after cessation of treatment with B 663 .

On the face, which was generally affected more than the trunk, it was the sites that were diffusely infiltrated or nodulated that became hyperpigmented most rapidly. The prominent areas, such as the superciliary ridges, the cheeks, the nose-tip, the chin, and the ears became darker than the rest of the face in several instances, whether or not they were the seat of obvious lepromatous infiltration.

On the trunk and limbs, the darkening was generalized, diffuse and symmetrical. Discrete lesions were usually, but not invariably, picked out by the hyperpigmentation. In the two patients with borderline leprosy, the skin just beyond the limits of the lesions was darker and more purplish than either the lesion itself or the surrounding skin. In some patients, the dorsa of the hands, and to a less extent the dorsa of the feet, became much darker than the rest of the skin. In one patient, the dorsal skin in the neighbourhood of the interphalangeal and metacarpophalangeal joints became black; in another the left hand was much blacker than the right; in yet another, one side of the nose was blacker than the other.

The conjunctivae, formerly but slightly pink, became muddy, and irregularly and diffusely pigmented.

As the leprosy lesions responded to the drug, the nodules became smaller, to be eventually replaced by scar tissue, recognizable as shiny circular macules, jet black in colour.

The black coloration persisted longer after the drug was stopped than did the redness. After the lapse of two years, it had disappeared in all but two patients, who were by nature deeply pigmented.

During its gradual disappearance, the dark coloration persisted in some patients as circumscribed hyperpigmented areas reminiscent of the elements of a dapsone-induced fixed eruption (Browne I959, I 964) or the post-inflammatory hypermelanotic staining following lesions of erythema nodosum leprosum (Browne i 963).

A baby (aged I 5 months, and still breast-fed) of the first patient to be treated with B 663, became ruddy like its mother, and subsequently developed a generalized darkening of the skin. Both the ruddiness and the darkening disappeared completely. (This child had some hazy patches of the trunk and face while the mother was taking B 663. No $M$. leprae were found in smears taken from the lesions, but their appearance resembled the abacillary prelepromatous macules not uncommon in West Africa.)

The skin of a baby born by Caesarean section of a mother who had received six months' treatment with B 663 together with dapsone (the last dose of B 663 having been given six weeks before delivery), was generally pinkish at birth, and became deeply red within a few weeks. The mother's milk was decidedly yelloworange in colour. The child's skin then became blackish brown, that is, much darker than would be expected in comparison with the degree of pigmentation of the parents' skin. The reddish 
coloration was noticeable in the periorbital skin for about six months af ter birth, but the hyperpigmentation persisted for nearly two years before finally disappearing.

\section{H IS T O L O G Y}

\section{The red coloration}

Specimens of skin removed under local anaesthetic and embedded and processed in the usual way, appeared to be generally and diffusely stained a bright pink - as with eosin. Examination of sections showed that the subcutaneous fat was more deeply stained than the dermis or epidermis. In preparations fixed and cleared secundum artem, no microcrystals of B 663 were visible and no 'ghosts' left by dissolved crystals could be discerned in sections of ordinary thickness. In frozen sections, however, Shepard ( i 964) has noted some accumulation of crystals in the liver of mice on very high drug intake, with foci of macrophages in and around the crystals. Some macrophages may actually contain the dye (Chang, I 964). These findings are in accord with the earlier experimental work of Barry et al (1959).

\section{The black coloration}

Specimens of skin removed at a latter stage, when it was becoming black to the naked eye, revealed a varying microscopic pattern that could be generally correlated with the clinical appearances.

At first, there was a reduplication of the stratum germinativum, with a consequent increase both in the number of strata of pigmentbearing cells and in the density of the pigment.

Later, single cells and then groups of elongated melanocytes in the stratum germinativum could be seen to have ruptured on their deep aspects, releasing their melanin content into the papillary layer of the dermis. Amorphous masses of melanin of varying dimensions were not only scattered in the papillary layer (especially between the rete pegs) but were also seen lying free and surrounded by lymphocytes in the reticular layer. In some cases, amorphous melanin was distributed in streaks and interrupted bands extending from the disorganized and disrupted cells of the stratum germinativum right through the dermis, often enclosed in a cylinder of round cells. This focalization resembled somewhat the localization of the granuloma in tuberculoid leprosy. At the level of Unna's palisade layer, the melanin might be distributed in a line parallel to the surface of the skin. In other cases, the masses of melanin were all of similar size, and rounded, giving the impression of being enclosed within a limiting membrane.

The dispersal of particulate masses of melanin deep in the dermis would, in conjunction with the brown colour of the normally pigmented skin, give rise to the various shades of blue-grey and blackish-brown coloration observed clinically.

\section{ISCUSSION}

Unlike the reported findings in animals receiving isoniazid in addition to B 663 (Chang I 962), the appearance of the ruddiness and hyperpigmentation was not postponed in those patients taking another drug (dapsone, or dapsone and ditophal) in addition to $\mathrm{B} 663$, nor was the apparent intensity of the red or the black pigmentation modified.

The widespread staining of the tissues by the safranins and the presence of microcrystals of B 663 in macrophages as well as extracellularly, do not appear to induce any cellular response in the experimental animal beyond a slight foreign body reaction. No local or systemic toxic effects at comparable dosages have been observed, nor has any carcinogenic effect been seen on prolonged administration of the drug. With long-continued high doses, however, the drug accumulates in the liver, and the possibility of toxic manifestations must not be overlooked (Shepard r 964; Chang r 964).

That the hyperpigmentation may have a complex pathology, is suggested by the work of Knight (ig64) and Wertlake (1964), who investigated two Mexican patients under treatment with B 663 at the National Institute of Allergy and Infectious Diseases, Washington. These workers were unable to detect significant differences in the amount of melanin pigment present in sections of skin taken from various sites, and suggest that a variable pattern may exist.

The hyperpigmentation is similar in some respects to that reported by Lowe (1952) in breast-fed babies whose mothers were taking dapsone. Doull (I959) noticed a hyperpigmentation virtually confined to areas of lepro- 
matous infiltration in patients taking high doses of amodiaquin for long periods (Browne ig6i). Some patients develop a generalized hypermelanosis following treatment with dapsone (Browne I959, I964), but in very few is the hyperpigmentation limited to skin affected by discrete or diffuse lepromatous infiltration. The hyperpigmentation observed in these patients under treatment with B 663 is unlike both the diffuse fixed eruption resulting from hypersensitivity and a generalized post-inflammatory hypermelanosis.

In spite of the changes in skin colour which might be objectionable in the lighter-hued, it is reassuring to observe that both the ruddiness and the hyperpigmentation diminish after the cessation of treatment and eventually disappear completely.

\section{S U M M A R Y}

The clinical and histological features of the red and black pigmentation of the skin developing in the course of treatment of leprosy patients with B 663 are reviewed. The coloration per se, being transitory, should not constitute an insuperable cosmetic objection to the use of the drug, especially in patients living among a population showing some considerable range of skin pigmentation.

\section{A CKNOWLEDGEMENTS}

My thanks are due to Messrs J. R. Geigy, s.A., of Basle, for generous supplies of their product, B 663; to Dr R. C. Cochrane, of the Leprosy Research Unit, London, for help with histology; and to Dr S. O. Egwuatu, Chief Medical Officer, Ministry of Health, Eastern Nigeria, for permission to publish this article.

\section{References}

Allday, E. J. and Barnes, J. (I 952). Irish 7. med Sci., 42 I. BARRY, v. C. et al. (I 957). Nature, I79, IOI3.

BARRY, v. C. et al. (1959). Bull. Int. Union Tuberculosis, 29, 582 .

BARRY, v. c. et al. (1960). Irish 7. med. Sci., 345 .

Browne, s. G. (I 959). Trans. R. Soc. trop. Med. Hyg., 53, 495 .

BRowne, s. G. ( 96 I). Int. 7. Leprosy, 29, 107.

browne, s. G. (1 963). 7. chr. Dis., r6, 23.

Browne, s. G. (1964). Brit. med. 7., 2, I 041.

Browne, s. G. and hogerzeil, L. M. (1 962a) Leprosy Rev., 33, 6 .

Browne, s. G. and hogerzeil, L. m. ( 1962b) Leprosy Rev., 33, 182 .

Chang, y. т. (1962). Antimicrobial Agents and Chemotherapy, 294.

CHANG, Y. T. (1964). Personal communication.

CONAlty, m. L. and JAGKson, R. D. (ig62a) Brit. 7. exp. Path., 43, 650.

Conalty, M. L. and Jackson, R. D. ( 1962b). Annales Histochimie Supp., 7, 221.

DOUll, J. A . (1959). Int. 7. Leprosy, 27, 385.

KNIGHT, VERNON (I 964). Personal communication.

LOWE, J. (1 952). Leprosy Rev., 23, 22.

SHEPARD, C. C. ( 1964 ). Personal communication.

wertlake, P. T. (1964). Personal communication. 